\title{
Mechanistic and Structural Analyses of the Roles of Arg409 and Asp402 in the Reaction of the Flavoprotein Nitroalkane Oxidase ${ }^{\dagger}, \downarrow$
}

Paul F. Fitzpatrick, ${ }^{\S} \|, *$, Dragana M. Bozinovski, ${ }^{\S}$ Annie Héroux ${ }^{\perp}$ Patrick G. Shaw, ${ }^{+}$Michael P. Valley, ${ }^{\S}$ and Allen M. Orville ${ }^{\perp, *}$

${ }^{\S}$ Departments of Biochemistry and Biophysics and of ${ }^{\|}$Chemistry, Texas A\&M University, College Station, TX 77843-2128

${ }^{\perp}$ Department of Biology, Brookhaven National Laboratory, Upton, New York 11973
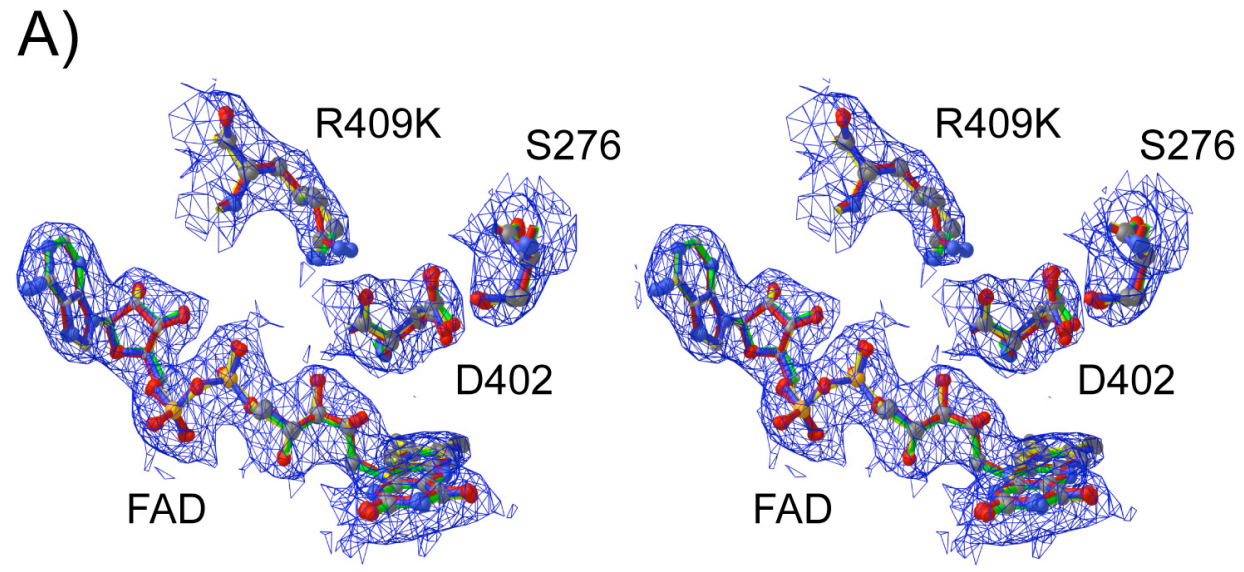

B)
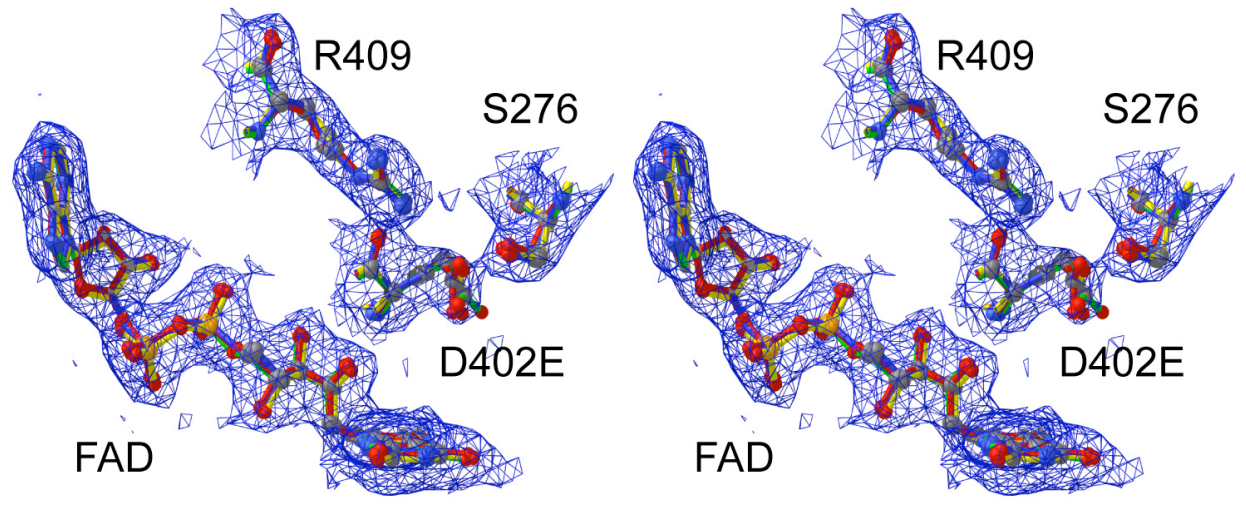

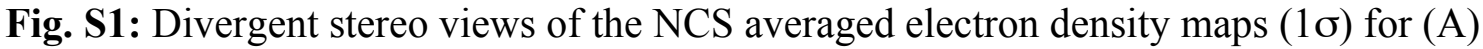
R409K and (B) D402E at $2.65 \AA$ and $2.4 \AA$ resolution, respectively. All four subunits are superimposed within the density with bonds shown in yellow, blue, green and red for subunits $\mathrm{A}, \mathrm{B}, \mathrm{C}$, and $\mathrm{D}$, respectively. Atoms are colored according to atom type. 
A)

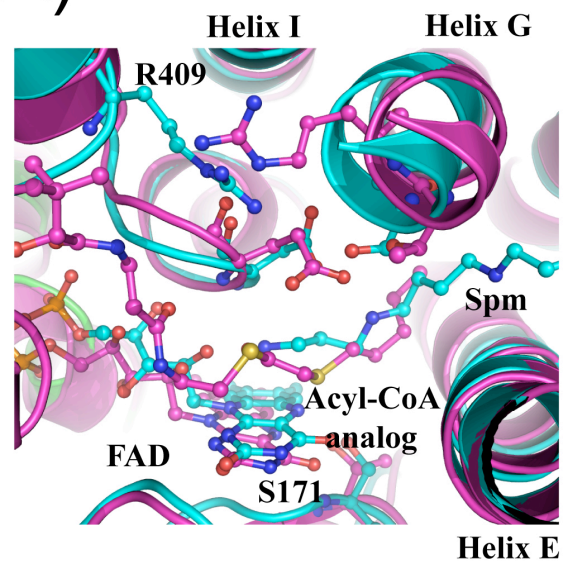

Helix I

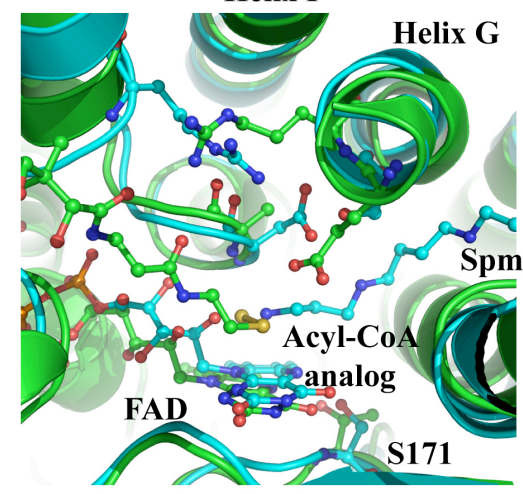

C)

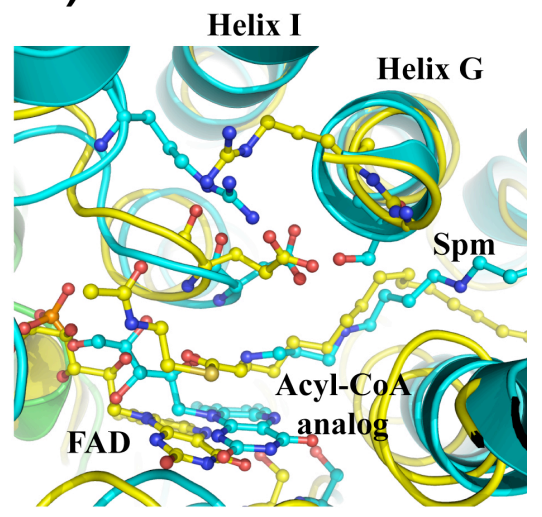

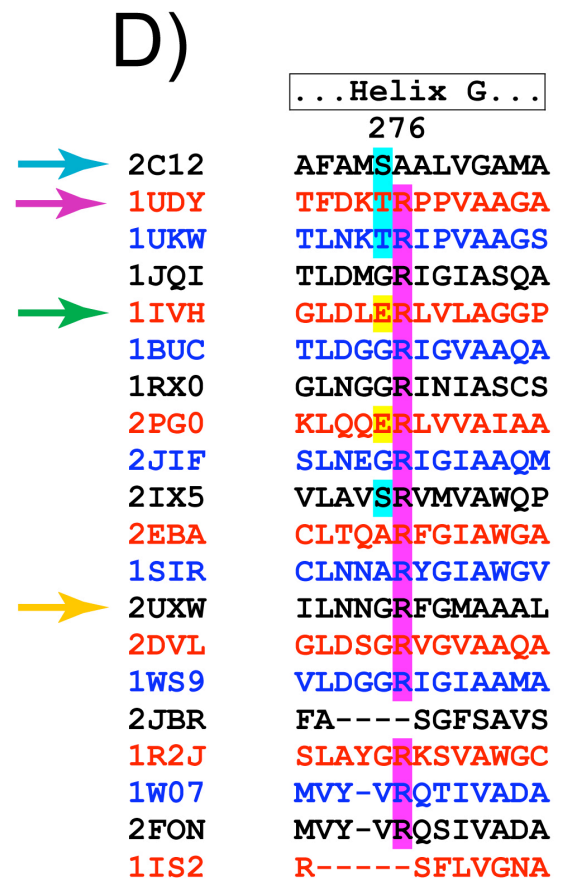

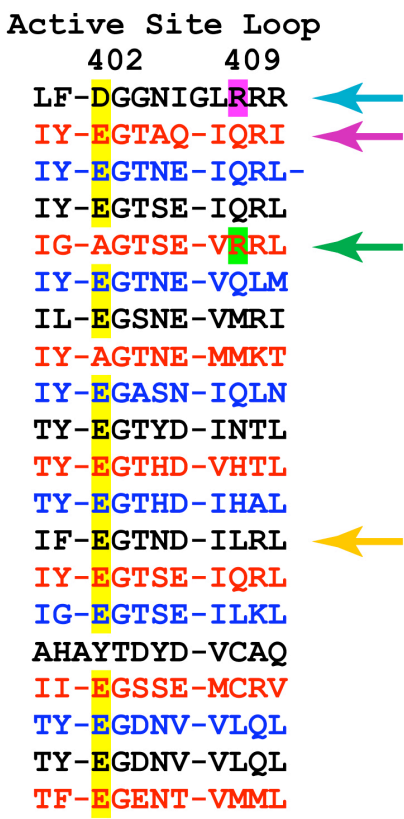

Fig. S2: Overlay of NAO and selected homologs. A) NAO (cyan, PDB code 2s12) superimposed with MCAD (magenta, PDB code 1udy); B) NAO superimposed with isovalerylCoA dehydrogenase (green, PDB code livh); C) NAO superimposed with human very long chain Acyl-CoA dehydrogenase (yellow. PDB code 2uxw). Shaded boxes correspond to residues in NAO that occupy the equivalent 3-dimensional space as Ser276 (cyan), Arg409 (magenta), Lys359 (green) and the active site base Asp402 (yellow). D) The secondary structure alignment (from the SSM web-server) for 20 homologs in the active site region of NAO illustrated in parts A-C. The numbering scheme corresponds to that of NAO (2C12). The full PDB titles are given below. 
2C12:A

1UDY:A

IUKW:A

1JQI:A

1IVH:A

1BUC:A

1RX0:A

2PG0:A

2JIF:A

2IX5:A

2EBA:A

1SIR:A

2UXW:A

2DVL:A

1WS9:A

2JBR:A

1R2J:A

1W07:A

2FON:A

1IS2:A
Crystal Structure of Nitroalkane Oxidase in Complex with Spermine, A Competitive Inhibitor Medium-Chain Acyl-CoA Dehydrogenase with 3-Thiaoctanoyl-CoA

Crystal structure of medium-chain acyl-CoA dehydrogenase from Thermus thermophilus HB8 Crystal Structure of Rat Short Chain Acyl-CoA Dehydrogenase Complexed with Acetoacetyl-CoA Structure of Human Isovaleryl-CoA Dehydrogenase: Structural Basis for Substrate Specificity Three-Dimensional Structure of Butyryl-CoA Dehydrogenase from Megasphaera elsdenii Crystal structure of isobutyryl-CoA dehydrogenase complexed with substrate/ligand Crystal structure of acyl-CoA dehydrogenase from Geobacillus kaustophilus Structure Of Human Short-Branched Chain Acyl-CoA Dehydrogenase (ACADSB) Short Chain Specific Acyl-CoA Oxidase from Arabidopsis thaliana, Acx4 in Complex with Acetoacetyl-CoA Crystal structure of the putative glutaryl-CoA dehydrogenase from Thermus thermophilus

The Crystal Structure and Mechanism of Human Glutaryl-CoA Dehydrogenase

Crystal Structure of Human Very Long Chain Acyl-CoA Dehydrogenase (ACADVL)

Crystal structure of project TT0160 from Thermus thermophilus HB8

Crystal structure of project ID TT0172 from Thermus thermophilus HB8

Monooxygenase Component of $p$-Hydroxyphenylacetate Hydroxylase from Acinetobacter baumanni

FkbI for Biosynthesis of Methoxymalonyl Extender Unit of Fk520 Polyketide Immunosuppresant Arabidopsis thaliana Acyl-Coa Oxidase 1

X-ray crystal structure of LeACX1, an acyl-CoA oxidase from Lycopersicon esculentum (tomato)

Crystal Structure of Peroxisomal Acyl-CoA Oxidase-II from Rat Liver 